\title{
THE RECTIFYING DEVELOPABLE AND RECTIFYING GAUSSIAN SURFACE OF CURVES IN PSEUDO-GALILEAN GEOMETRY
}

\author{
BY \\ TEVFIK ŞAHIN and MURTEZA YILMAZ
}

\begin{abstract}
In this paper, we give the classification of the singularities of pseudoGalilean spherical Darboux image, rectifying Gaussian surface and the rectifying developable of curve in pseudo-Galilean space. We also establish the relationships between the singularities and geometric invariants of curves which are deeply related to its order of contact with pseudo-Galilean helices.
\end{abstract}

Mathematics Subject Classification 2010: 58K05, 53A35.

Key words: height function, distance function, singularities, pseudo-Galilean space.

\section{Introduction}

Singularity theory, being a direct descendant of differential calculus, is certain to have a great deal of interest to say about geometry and therefore about all the branches of mathematics, physics and other disciplines where the geometrical spirit is a guiding light.

Several geometers were interested in studying the singularities and generic differential geometry in Euclidean Space $[1,2-4,12,13]$. The main point of studying singularity is defining real-valued functions such as squareddistance function and height function which are defined on a curve or on a surface. The classical invariants of extrinsic differential geometry can be treated as singularities of these two functions. Also, some good approximations to singularity theory in affine geometry can be found in $[9,10]$ and Minkowski space can be found in $[5,15]$. 
Besides Euclidean Geometry, a range of new types of geometries have been invented and developed in the last two centuries. They can be introduced in a variety of ways. One possible way is through projective manner, where one can express metric properties through projective relations. Among these geometries, there is also pseudo-Galilean geometry which is our matter in this paper.

We will introduce the notion of pseudo-Galilean distance functions on space curves in $G_{3}^{1}$, pseudo-Galilean space. This function is quite useful for the study of singularities of rectifying developable of space curves in $G_{3}^{1}$. We also introduce the notion of pseudo-Galilean height functions on space curves in $G_{3}^{1}$, which induce the notion of rectifying Gaussian surface and spherical Darboux images and these singularities are deeply related to the geometry of pseudo-Galilean spherical tangential images of curves in pseudo-Galilean 3-space.

As a consequence, we establish the relationships between the singularities of the above three subjects and differential geometric invariants of the curve under the action of pseudo-Galilean group as applications of ordinary techniques of singularity theory for the above functions. As the spheres degenerate in the pseudo-Galilean space it is not obvious in the first place that this concept would provide geometric results. However, we can prove that these functions applied to curves lead to geometric characterisations even in pseudo-Galilean space. For the basic notions in pseudo-Galilean geometry, see $[6,17]$.

The main result in this paper is Theorem 1 . We describe the geometric meaning of Theorem 1 in $\S 3.2$ and 3.3. The basic techniques used depend heavily on those in the book of BRUCE and GIBLIN [4].

\section{Preliminaries on pseudo-Galilean geometry}

The pseudo-Galilean space $G_{3}^{1}$ is a Cayley-Klein space equipped with the projective metric of signature $(0,0,+,-)$, as in [11]. The absolute of the pseudo-Galilean geometry is an ordered triple $\{w, f, I\}$, where $w$ is the ideal (absolute) plane, $f$ is the line (absolute line) in $w$ and $I$ is the fixed hyperbolic involution of points of $f$.

Let $P$ be any point of $\mathbb{R}^{3}$ with affine coordinates $(x, y, z)$. Write $(x, y, z)$ as $\left(\frac{X_{1}}{X_{0}}, \frac{X_{2}}{X_{0}}, \frac{X_{3}}{X_{0}}\right)$, where $X_{0}$ is some common deminator. Call $\left(X_{0}, X_{1}, X_{2}, X_{3}\right)$ the homogeneous coordinates of $P$. Thus, the homogeneous coordinates $\left(X_{0}: X_{1}: X_{2}: X_{3}\right)$ and $\rho\left(X_{0}: X_{1}: X_{2}: X_{3}\right)$ refer to the same point, for 
all $\rho \in \mathbb{R}-\{0\}$. We can now introduce homogeneous coordinates in $G_{3}^{1}$ in such a way that the absolute plane $w$ is given by $X_{0}=0$, the absolute line $f$ by $X_{0}=X_{1}=0$ and the hyberbolic involution $I$ by

$$
\left(0: 0: X_{2}: X_{3}\right) \rightarrow\left(0: 0: X_{3}: X_{2}\right)
$$

In affine coordinates, the distance between the points $P_{i}\left(x_{i}, y_{i}, z_{i}\right), i=$ 1,2 , is defined by

$$
d\left(P_{1}, P_{2}\right)=\left\{\begin{array}{ll}
\left|x_{2}-x_{1}\right|, & \text { if } x_{1} \neq x_{2} \\
\sqrt{\left|\left(y_{2}-y_{1}\right)^{2}-\left(z_{2}-z_{1}\right)^{2}\right|} & \text { if } x_{1}=x_{2}
\end{array} .\right.
$$

In the nonhomogeneous coordinates the isometries group $B_{6}$ has the form:

$$
\begin{aligned}
& \bar{x}=a+x, \\
& \bar{y}=b+c x+y \cosh \varphi+z \sinh \varphi \\
& \bar{z}=d+e x+y \sinh \varphi+z \cosh \varphi
\end{aligned}
$$

where $a, b, c, d, e$ and $\varphi$ are real numbers. The group of motions of $G_{3}^{1}$ is a six-parameter group $[6,15,16]$.

It leaves invariant the pseudo-Galilean norm of the vector $A(x, y, z)$ defined by

$$
\|A\|_{p G}=\left\{\begin{array}{ll}
|x|, & \text { if } x \neq 0 \\
\sqrt{\left|y^{2}-z^{2}\right|,} & \text { if } x=0
\end{array} .\right.
$$

A vector $A(x, y, z)$ is said to be non-isotropic if $x \neq 0$. All unit non-isotropic vectors are of the form $(1, y, z)$. For isotropic vectors $x=0$ holds and four types of isotropic vectors: spacelike $\left(y^{2}-z^{2}>0\right)$, timelike $\left(y^{2}-z^{2}<0\right)$ and two types of lightlike vectors $(y= \pm z)$.

The scalar product of two vectors $\mathbf{a}=\left(a_{1}, a_{2}, a_{3}\right)$ and $\mathbf{b}=\left(b_{1}, b_{2}, b_{3}\right)$ in $G_{3}^{1}$ is defined by

$$
\mathbf{a} \cdot p G \mathbf{b}=\left\{\begin{array}{ll}
a_{1} b_{1}, & \text { if } a_{1} \neq 0 \text { or } b_{1} \neq 0 \\
a_{2} b_{2}-a_{3} b_{3}, & \text { if } a_{1}=0 \text { and } b_{1}=0
\end{array} .\right.
$$

If $\mathbf{a} \cdot{ }_{p G} \mathbf{b}=0$, then $\mathbf{a}$ and $\mathbf{b}$ are perpendicular. ( Of course every isotropic vector is perpendicular to every non-isotropic vector.) 
For a curve $\gamma: I \rightarrow G_{3}^{1}, I \subset \mathbb{R}$ parametrized by the invariant parameter $s=x$, given in the coordinate form

$$
\gamma(x)=(x, y(x), z(x)),
$$

the curvature $\kappa(x)$ and the torsion $\tau(x)$ are defined by

$$
\kappa(x)=\sqrt{\left|y^{\prime \prime}(x)^{2}-z^{\prime \prime}(x)^{2}\right|}, \quad \tau(x)=\frac{\operatorname{det}\left(\gamma^{\prime}(x), \gamma^{\prime \prime}(x), \gamma^{\prime \prime \prime}(x)\right)}{\kappa^{2}(x)}
$$

and the associated moving trihedron is given by

$$
\begin{aligned}
t(x) & =\gamma^{\prime}(x)=\left(1, y^{\prime}(x), z^{\prime}(x)\right), \\
n(x) & =\frac{1}{\kappa(x)}\left(0, y^{\prime \prime}(x), z^{\prime \prime}(x)\right), \\
b(x) & =\frac{1}{\kappa(x)}\left(0, \varepsilon z^{\prime \prime}(x), \varepsilon y^{\prime \prime}(x)\right),
\end{aligned}
$$

where $\varepsilon= \pm 1$, chosen by criterion $\operatorname{det}(t, n, b)=1$, that means

$$
\left|y^{\prime \prime}(x)^{2}-z^{\prime \prime}(x)^{2}\right|=\varepsilon\left(y^{\prime \prime}(x)^{2}-z^{\prime \prime}(x)^{2}\right) .
$$

The curve $\gamma$ is time-like (resp. space-like) if $n(x)$ is a space-like (resp. time-like) vector. The principal normal vector or simply normal is spacelike if $\varepsilon=+1$ and time-like if $\varepsilon=-1$. The vectors $t(x), n(x)$ and $b(x)$ are called the vectors of the tangent, principal normal and the binormal line, respectively [8]. Therefore, the Frenet-Serret formulas can be written in matrix notation as

$$
\left[\begin{array}{c}
t \\
n \\
b
\end{array}\right]^{\prime}=\left[\begin{array}{lll}
0 & \kappa & 0 \\
0 & 0 & \tau \\
0 & \tau & 0
\end{array}\right]\left[\begin{array}{l}
t \\
n \\
b
\end{array}\right]
$$

From (4) and (5) one gets an important relation

$$
\gamma^{\prime \prime \prime}(x)=\kappa^{\prime}(x) n(x)+\kappa(x) \tau(x) b(x)([8]) .
$$

For any curve $\gamma: I \rightarrow G_{3}^{1}$, we call $D(x)=-\tau(x) t(x)+\kappa(x) b(x)$ a Darboux vector of $\gamma([6])$. By using the Darboux vector, Frenet-Serret formulas can be rewritten as follows:

$$
\begin{aligned}
t^{\prime}(x) & =D(x) \times_{p G} t(x), \\
n^{\prime}(x) & =D(x) \times_{p G} n(x), \\
b^{\prime}(x) & =D(x) \times_{p G} b(x),
\end{aligned}
$$


where the pseudo-Galilean cross product $\times_{p G}$ is defined by

$$
\mathbf{a} \times_{p G} \mathbf{b}=\left|\begin{array}{ccc}
0 & e_{2} & -e_{3} \\
a_{1} & a_{2} & a_{3} \\
b_{1} & b_{2} & b_{3}
\end{array}\right|
$$

for $\mathbf{a}=\left(a_{1}, a_{2}, a_{3}\right)$ and $\mathbf{b}=\left(b_{1}, b_{2}, b_{3}\right)$. The pseudo-Galilean Sphere $S_{p G}^{2}$ is defined by $S_{p G}^{2}=\left\{(x, y, z) \in G_{3}^{1}|| x-x_{0} \mid=r\right\}$.

For more on Galilean and pseudo-Galilean geometry, one can refer to $[6-8,16,17]$ and references therein.

\section{Singularities of some functions in pseudo-Galilean geometry}

We define a vector $\widetilde{D}(x)=\left(-\frac{\tau}{\kappa}\right)(x) t(x)+b(x)$ and call it a modified Darboux vector along $\gamma$. We also define a spherical curve $d: I \rightarrow S_{p G}^{2}$ by $d(x)=\frac{D(x)}{\|D(x)\|_{p G}}$ and surfaces

$$
\begin{aligned}
& R G(\gamma)=\{u t(x)+b(x) \mid u \in \mathbb{R}, x \in I\} \\
& R D(\gamma)=\{\gamma(x)+u \widetilde{D}(x) \mid u \in \mathbb{R}\} .
\end{aligned}
$$

We call the image of $d$ the pseudo-Galilean spherical Darboux image, the surface $R G(\gamma)$ the rectifying Gaussian surface and the surface $R D(\gamma)$ the rectifying developable of $\gamma$.

Theorem 1. Let $\gamma: I \rightarrow G_{3}^{1}$ be a unit speed curve. Then:

(1) The pseudo-Galilean spherical Darboux image is locally diffeomorphic to the ordinary cusp $C$ at $d\left(x_{0}\right)$ if and if only $\left(\frac{\tau}{\kappa}\right)^{\prime}\left(x_{0}\right)=0$ and $\left(\frac{\tau}{\kappa}\right)^{\prime \prime}\left(x_{0}\right) \neq 0$.

(2) (a) The rectifying Gaussian surface is locally diffeomorphic to the cuspidal edge $C \times \mathbb{R}$ at $u_{0} t\left(x_{0}\right)+b\left(x_{0}\right)$ if and if only $u_{0}=\left(\frac{\tau}{\kappa}\right)\left(x_{0}\right)$ and $\left(\frac{\tau}{\kappa}\right)^{\prime}\left(x_{0}\right) \neq 0$.

(b) The rectifying Gaussian surface is locally diffeomorphic to the swallowtail $S W$ at $u_{0} t\left(x_{0}\right)+b\left(x_{0}\right)$ if and if only $u_{0}=\left(\frac{\tau}{\kappa}\right)\left(x_{0}\right),\left(\frac{\tau}{\kappa}\right)^{\prime}\left(x_{0}\right)=0$ and $\left(\frac{\tau}{\kappa}\right)^{\prime \prime}\left(x_{0}\right) \neq 0$.

(3) (a) The rectifying developable is locally diffeomorphic to the cuspidal edge $C \times \mathbb{R}$ at $\gamma\left(x_{0}\right)+u_{0} \widetilde{D}\left(x_{0}\right)$ if and if only $\left(\frac{\tau}{\kappa}\right)^{\prime}\left(x_{0}\right) \neq 0, u_{0}=\frac{1}{\left(\frac{\tau}{\kappa}\right)^{\prime}\left(x_{0}\right)}$ and $\left(\frac{\tau}{\kappa}\right)^{\prime \prime}\left(x_{0}\right) \neq 0$. 
(b) The rectifying developable is locally diffeomorphic to the swallowtail $S W$ at $\gamma\left(x_{0}\right)+\widetilde{u_{0} D}\left(x_{0}\right)$ if and if only $\left(\frac{\tau}{\kappa}\right)^{\prime}\left(x_{0}\right) \neq 0, u_{0}=\frac{1}{\left(\frac{\tau}{\kappa}\right)^{\prime}\left(x_{0}\right)}$ ,$\left(\frac{\tau}{\kappa}\right)^{\prime \prime}\left(x_{0}\right)=0$ and $\left(\frac{\tau}{\kappa}\right)^{\prime \prime \prime}\left(x_{0}\right) \neq 0$.

Here, $C=\left\{\left(x_{1}, x_{2}\right): x_{1}^{2}=x_{2}^{3}\right\}$ is ordinary cusp and $S W=\left\{\left(x_{1}, x_{2}, x_{3}\right):\right.$ $\left.x_{1}=3 u^{4}+u^{2} v, x_{2}=4 u^{3}+2 u v, x_{3}=v\right\}$ is the swallowtail.
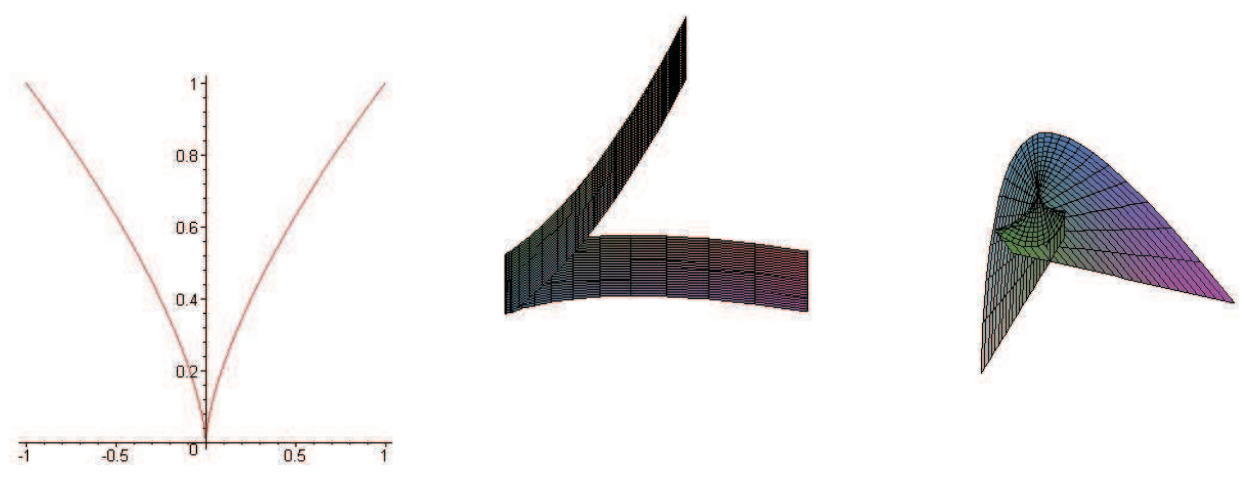

Figure 1: The cusp curve, The cuspidal edge and The swallowtail surface

The main aim of this paper is proving this Theorem 1. For this issue, we will study the singularities of height function, distance function in pseudoGalilean space in section 3.1. Also, since we need the unfoldings of functions in $G_{3}^{1}$, we describe the content of them in section 3.3. As we study in 3dimensional space, we need to look beyond $A_{4}$-singularities which is defined in section 3.3. Therefore, we will compute higher order derivatives of height function and distance function in Proposition 2 and Proposition 3.

\subsection{Families of smooth functions on a space curve in pseudo- Galilean geometry}

In this section two different families of function on a space curve and surface will be defined which are useful for the study of singularities of pseudo-Galilean spherical Darboux image, the rectifying Gaussian surface and the rectifying developable surface. Let $\gamma: I \rightarrow G_{3}^{1}$ be a unit speed curve with $\kappa(x) \neq 0$. We will assume that $\tau(x) \neq 0$ throughout this paper, because we only consider the singularities of rectifying developable of $\gamma$. 


\subsubsection{Height function in pseudo-Galilean space}

We introduce the notion of pseudo-Galilean height functions on space curves in $G_{3}^{1}$, which induce the notion of rectifying Gaussian surface and spherical Darboux images and these singularities are deeply related to the geometry of pseudo-Galilean spherical tangential images of curves in pseudoGalilean 3-space. We now define a two-parameter family of smooth functions on $I$ :

$$
F_{h}: I \times S_{p G}^{2} \rightarrow \mathbb{R}
$$

by $F_{h}(x, \mathbf{v})=|t(x) \quad n(x) \quad \mathbf{v}|$. Here, $\left|\begin{array}{lll}\mathbf{a} \quad \mathbf{b} & \mathbf{c}\end{array}\right|$ denotes the determinant of the matrix ( $\mathbf{a} \mathbf{b} \mathbf{c}$ ) and the vectors $t(x), n(x)$ are the vectors of the tangent and principal normal, respectively [8]. We call $F_{h}$ a pseudo-Galilean height function on $\gamma$. Denote $f_{h v}(x)=F_{h}(x, \mathbf{v})$ for any $\forall \mathbf{v} \in S_{p G}^{2}$. Then, we have the following proposition.

Proposition 2. Let $\gamma: I \rightarrow G_{3}^{1}$ be a unit speed curve with $\kappa(x) \neq 0$ and $\tau(x) \neq 0$. Then:

(1) $f_{h v}^{\prime}(x)=0$ if and only if there exist real numbers $\lambda, \mu \in \mathbb{R}$, such that $\mathbf{v}=\lambda t(x)+\mu b(x),|\lambda|= \pm 1$.

(2) $f_{h v}^{\prime}(x)=f_{h v}^{\prime \prime}(x)=0$ if and only if

$$
\mathbf{v}=\mp\left(\frac{\kappa}{\tau}\right)(x)\left(\left(-\frac{\tau}{\kappa}\right)(x) t(x)+b(x)\right) .
$$

(3) $f_{h v}^{\prime}(x)=f_{h v}^{\prime \prime}(x)=f_{h v}^{\prime \prime \prime}(x)=0$ if and only if

$$
\mathbf{v}=\mp\left(\frac{\kappa}{\tau}\right)(x)\left(\left(-\frac{\tau}{\kappa}\right)(x) t(x)+b(x)\right),\left(\frac{\tau}{\kappa}\right)^{\prime}(x)=0 .
$$

(4) $f_{h v}^{\prime}(x)=f_{h v}^{\prime \prime}(x)=f_{h v}^{\prime \prime \prime}(x)=f_{h v}^{(4)}(x)=0$ if and only if

$$
\mathbf{v}=\mp\left(\frac{\kappa}{\tau}\right)(x)\left(\left(-\frac{\tau}{\kappa}\right)(x) t(x)+b(x)\right),\left(\frac{\tau}{\kappa}\right)^{\prime}(x)=\left(\frac{\tau}{\kappa}\right)^{\prime \prime}(x)=0 .
$$

(5) $f_{h v}^{\prime}(x)=f_{h v}^{\prime \prime}(x)=f_{h v}^{\prime \prime \prime}(x)=f_{h v}^{(4)}(x)=f_{h v}^{(5)}(x)=0$ if and only if $\mathbf{v}=\mp\left(\frac{\kappa}{\tau}\right)(x)\left(\left(-\frac{\tau}{\kappa}\right)(x) t(x)+b(x)\right),\left(\frac{\tau}{\kappa}\right)^{\prime}(x)=\left(\frac{\tau}{\kappa}\right)^{\prime \prime}(x)=\left(\frac{\tau}{\kappa}\right)^{\prime \prime \prime}(x)=0$. 
Proof. By the Frenet-Serret formula, we have the following calculation:

(i) $\quad f_{h v}^{\prime}(x)=\tau(x)|t(x) b(x) \mathbf{v}|$

(ii) $\quad f_{h v}^{\prime \prime}(x)=\tau^{\prime}(x)|t(x) b(x) \mathbf{v}|+\kappa(x) \tau(x)|n(x) b(x) \mathbf{v}|+\tau^{2}(x)|t(x) n(x) \mathbf{v}|$.

(iii) $\quad f_{h v}^{\prime \prime \prime}(x)=\left(\tau^{\prime \prime}(x)+\tau^{3}(x)\right)|t(x) b(x) \mathbf{v}|+\left(2 \kappa(x) \tau^{\prime}(x)\right.$

$$
\left.+\kappa^{\prime}(x) \tau(x)\right)|n(x) b(x) \mathbf{v}|+3 \tau(x) \tau^{\prime}(x)|t(x) n(x) \mathbf{v}| .
$$

$$
\begin{aligned}
(i v) \quad f_{h v}^{(4)}(x) & =\left(\tau^{\prime \prime \prime}(x)+6 \tau^{2}(x) \tau^{\prime}(x)\right)|t(x) b(x) \mathbf{v}|+\left(3 \kappa(x) \tau^{\prime \prime}(x)\right. \\
& \left.+\kappa(x) \tau^{3}(x)+3 \kappa^{\prime}(x) \tau^{\prime}(x)+\kappa^{\prime \prime}(x) \tau(x)\right)|n(x) b(x) \mathbf{v}| \\
& +\left(4 \tau(x) \tau^{\prime \prime}(x)+\tau^{4}(x)+3 \tau^{\prime 2}(x)\right)|t(x) n(x) \mathbf{v}| . \\
(v) \quad f_{h v}^{(5)}(x) & =\left(\tau^{(4)}(x)+15 \tau(x) \tau^{\prime 2}(x)+10 \tau^{2}(x) \tau^{\prime \prime}(x)+\tau^{5}(x)\right)|t(x) b(x) \mathbf{v}| \\
& +\left(6 \kappa^{\prime}(x) \tau^{\prime \prime}(x)+4 \kappa(x) \tau^{\prime \prime \prime}(x)+4 \kappa^{\prime \prime}(x) \tau^{\prime}(x)\right. \\
& \left.+9 \kappa(x) \tau^{2}(x) \tau^{\prime}(x)+\kappa^{\prime \prime \prime}(x) \tau(x)+\kappa^{\prime}(x) \tau^{3}(x)\right)|n(x) b(x) \mathbf{v}| \\
& +\left(10 \tau^{3}(x) \tau^{\prime}(x)+10 \tau^{\prime}(x) \tau^{\prime \prime}(x)+5 \tau(x) \tau^{\prime \prime \prime}(x)\right)|t(x) n(x) \mathbf{v}|
\end{aligned}
$$

The assertion (1) is trivial by the formula (i). By the assumption that $\mathbf{v} \in S_{p G}^{2}$, we have $\mathbf{v}= \pm t(x)+\mu b(x)$.

(2) By using (1) in (ii), we get $\mu=\mp\left(\frac{\kappa}{\tau}\right)(x)$. Therefore we have $\mathbf{v}=\mp\left(\frac{\kappa}{\tau}\right)(x)\left(\left(-\frac{\tau}{\kappa}\right)(x) t(x)+b(x)\right)$.

(3) By using (2) in (iii), then we get $\left(\frac{\tau}{\kappa}\right)^{\prime}(x)=0$ and the assertion (3) follows.

(4) If we substitute (3) into (iv) and use $\left(\frac{\tau}{\kappa}\right)^{\prime}(x)=0$, then we get $\left(\frac{\tau}{\kappa}\right)^{\prime \prime}(x)=0$.

(5) By similar calculation, we have the assertion (5).

\subsubsection{Distance function in pseudo-Galilean space}

We will introduce the notion of pseudo-Galilean distance functions on space curves in $G_{3}^{1}$, pseudo-Galilean space. This function is quite useful for the study of singularities of rectifying developable of space curves in $G_{3}^{1}$. We now define a three-parameter family of smooth functions on $I$ :

$$
F_{d}: I \times G_{3}^{1} \rightarrow \mathbb{R}
$$

by $F_{d}(x, \mathbf{u})=|t(x) \quad n(x) \quad \gamma(x)-\mathbf{u}|$. We call $F_{d}$ a pseudo-Galilean distance function on $\gamma$. Denote $f_{d u}(x)=F_{d}(x, \mathbf{u})$ for any $\mathbf{u} \in G_{3}^{1}$. Then, we have the following proposition. 
Proposition 3. Let $\gamma: I \rightarrow G_{3}^{1}$ be a unit speed curve with $\kappa(x) \neq 0$ and $\tau(x) \neq 0$. Then:

(1) $f_{d u}^{\prime}(x)=0$ if and only if there exist real numbers $\lambda, \mu \in \mathbb{R}$, such that $\gamma(x)-\mathbf{u}=\lambda t(x)+\mu b(x)$.

(2) $f_{d u}^{\prime}(x)=f_{d u}^{\prime \prime}(x)=0$ if and only if $\gamma(x)-\mathbf{u}=\mu\left(\left(-\frac{\tau}{\kappa}\right)(x) t(x)+b(x)\right)$.

(3) $f_{d u}^{\prime}(x)=f_{d u}^{\prime \prime}(x)=f_{d u}^{\prime \prime \prime}(x)=0$ if and only if

$$
\gamma(x)-\mathbf{u}=\frac{-1}{\left(\frac{\tau}{\kappa}\right)^{\prime}(x)}\left(\left(-\frac{\tau}{\kappa}\right)(x) t(x)+b(x)\right),\left(\frac{\tau}{\kappa}\right)^{\prime}(x) \neq 0 .
$$

(4) $f_{d u}^{\prime}(x)=f_{d u}^{\prime \prime}(x)=f_{d u}^{\prime \prime \prime}(x)=f_{d u}^{(4)}(x)=0$ if and only if

$\gamma(x)-\mathbf{u}=\frac{-1}{\left(\frac{\tau}{\kappa}\right)^{\prime}(x)}\left(\left(-\frac{\tau}{\kappa}\right)(x) t(x)+b(x)\right),\left(\frac{\tau}{\kappa}\right)^{\prime}(x) \neq 0,\left(\frac{\tau}{\kappa}\right)^{\prime \prime}(x)=0$.

Proof. By the Frenet-Serret formula, we have the following calculation:

(i) $\quad f_{d u}^{\prime}(x)=\tau(x)|t(x) b(x) \gamma(x)-\mathbf{u}|$.

(ii) $\quad f_{d u}^{\prime \prime}(x)=\tau^{\prime}(x)|t(x) b(x) \gamma(x)-\mathbf{u}|$

$$
+\kappa(x) \tau(x)|n(x) b(x) \gamma(x)-\mathbf{u}|+\tau^{2}(x)|t(x) n(x) \gamma(x)-\mathbf{u}|
$$

(iii) $\quad f_{d u}^{\prime \prime \prime}(x)=\left(\tau^{\prime \prime}(x)+\tau^{3}(x)\right)|t(x) b(x) \gamma(x)-\mathbf{u}|$

$$
\begin{aligned}
& +\left(2 \kappa(x) \tau^{\prime}(x)+\kappa^{\prime}(x) \tau(x)\right)|n(x) b(x) \gamma(x)-\mathbf{u}| \\
& +3 \tau(x) \tau^{\prime}(x)|t(x) n(x) \gamma(x)-\mathbf{u}|+\kappa(x) \tau(x)
\end{aligned}
$$

(iv) $f_{d u}^{(4)}(x)=\left(\tau^{\prime \prime \prime}(x)+6 \tau^{2}(x) \tau^{\prime}(x)\right)|t(x) b(x) \gamma(x)-\mathbf{u}|$

$$
\begin{aligned}
& +\left(3 \kappa(x) \tau^{\prime \prime}(x)+\kappa(x) \tau^{3}(x)+3 \kappa^{\prime}(x) \tau^{\prime}(x)\right. \\
& \left.+\kappa^{\prime \prime}(x) \tau(x)\right)|n(x) b(x) \gamma(x)-\mathbf{u}| \\
& +\left(4 \tau(x) \tau^{\prime \prime}(x)+\tau^{4}(x)+3 \tau^{\prime 2}(x)\right)|t(x) n(x) \gamma(x)-\mathbf{u}| \\
& +2 \kappa^{\prime}(x) \tau(x)+3 \kappa(x) \tau^{\prime}(x) .
\end{aligned}
$$

(1) The assertion follows from the above formula (i). Then, we have $\gamma(x)-\mathbf{u}=\lambda t(x)+\mu b(x)$.

(2) By using (1) in (ii), we get $\lambda=\left(-\frac{\tau}{\kappa}\right)(x) \mu$. Therefore we have $\gamma(x)-\mathbf{u}=\mu\left(\left(-\frac{\tau}{\kappa}\right)(x) t(x)+b(x)\right)$.

(3) If we now substitute the formula $\gamma(x)-\mathbf{u}=\mu\left(\left(-\frac{\tau}{\kappa}\right)(x) t(x)+b(x)\right)$ into (iii), then we have $\mu=\frac{-1}{\left(\frac{\tau}{\kappa}\right)^{\prime}(x)}$ and $\left(\frac{\tau}{\kappa}\right)^{\prime}(x) \neq 0$.

(4) By using (3) in (iv), we get $\left(\frac{\tau}{\kappa}\right)^{\prime \prime}(x)=0$. 
3.2. Pseudo-Galilean helices and the tangent indicatrix of a curve

We study the geometric properties of the rectifying developable, the pseudoGalilean spherical Darboux image and rectifying Gaussian surface of space curves in $G_{3}^{1}$. By the propositions in the last section, one can recognize that the function $\left(\frac{\kappa}{\tau}\right)^{\prime}(x)$ and the modified Darboux vector $\left(-\frac{\tau}{\kappa}\right)(x) t(x)+b(x)$ are important subjects. If $\left(\frac{\kappa}{\tau}\right)(x) \equiv c$ (constant) then the curve $\gamma(x)$ has been classically known as a helix in pseudo-Galilean space. Pseudo-Galilean cycle is the only curves of constant curvature in plane [6]. For a unit speed regular curve $\gamma(x)$ has tangent curve $\sigma: I \rightarrow S_{p G}^{2}, \sigma(x)=t(x)$ is called the pseudo-Galilean spherical tangential image of $\gamma(x)$.

Proposition 4. Let $\gamma: I \rightarrow G_{3}^{1}$ be a unit speed regular curve. Then $\gamma(x)$ is a helix if and only if the modified Darboux vector $\widetilde{D}(x)$ is a constant vector. In this case we have the following assertions:

(1) The Galilean spherical tangential image $\sigma(x)$ of $\gamma(x)$ is a cycle on the unit pseudo-Galilean sphere $S_{p G}^{2}$.

(2) The rectifying developable of $\gamma(x)$ is a cylindrical surface given by $\gamma(x)+$ ue, where $e=\widetilde{D}(x)$.

Proof. By the Frenet-Serret formulas, we can show that $\widetilde{D}^{\prime}(x)=$ $\left(-\frac{\tau}{\kappa}\right)^{\prime}(x) t(x)$. Therefore, $\gamma(x)$ is a helix if and only if $\widetilde{D}^{\prime}(x) \equiv 0$. This condition is equivalent to the condition that $\widetilde{D}(x)$ is a constant vector. In this case we have $\sigma(x)=t(x), \sigma^{\prime}(x)=\kappa(x) n(x), \sigma^{\prime \prime}(x)=\kappa^{\prime}(x) n(x)+$ $\kappa(x) \tau(x) b(x)$. The curvature of $\sigma(x)$ is $\kappa_{\sigma}(x)=\left(\frac{\tau}{\kappa}\right)(x)=$ constant. This means that the pseudo-Galilean spherical tangential image $\sigma(x)$ is a cycle on the unit pseudo-Galilean sphere $S_{p G}^{2}$. The assertion (2) is clear by definition.

The singularities of the pseudo-Galilean spherical Darboux image ( or, the rectifying Gaussian surface) describe how the shape of the curve $\gamma$ is similar to a helix. On the other hand, the singularities of the rectifying developable of $\gamma$ describe how the shape of the curve $\gamma$ is different from a helix.

\subsection{Unfoldings of functions by one-variable}

In this section, we will use some general results on singularity theory for families of function germs. Let $F:\left(I \times \mathbb{R}^{r},\left(x_{0}, w_{0}\right)\right) \rightarrow \mathbb{R}$ be a function germ. we call $F$ an r-parameter unfolding of $f$, where $f(x)=F_{w_{0}}\left(x, w_{0}\right)$. 
We say that $f$ has $A_{k}$-singularity at $x_{0}$ if $f^{(p)}\left(x_{0}\right)=0$ for all $1 \leq$ $p \leq k$ and $f^{(k+1)}\left(x_{0}\right) \neq 0$. We also say that $f$ has $A_{\geq k}-$ singularity at $x_{0}$ if $f^{(p)}\left(x_{0}\right)=0$ for all $1 \leq p \leq k$. Let $F$ be an unfolding of $f$ and $f(x)$ has $A_{k}-$ singularity $(k \geq 1)$ at $x_{0}$. Denote the $(k-1)$-jet of the partial derivative $\frac{\partial F}{\partial w_{i}}$ at $x_{0}$ by $J^{k-1}\left(\frac{\partial F}{\partial w_{i}}\left(x, w_{0}\right)\right)\left(x_{0}\right)=\sum_{j=1}^{k-1} \alpha_{i j} x^{j}$ for $i=1, \ldots, r$. Then $F$ is called $a(p)$-versal unfolding if the $(k-1) \times r$ matrix of coefficients $\left(\alpha_{i j}\right)$ has rank $k-1(k-1 \leq r)$. Under the same condition as above, $F$ is called a versal unfolding if the $k \times r$ matrix of coefficients $\left(\alpha_{0 i}, \alpha_{i j}\right)$ has rank $k(k \leq r)$, where $\alpha_{0 i}=\frac{\partial F}{\partial w_{i}}\left(x_{0}, w_{0}\right)$.

We now introduce important sets concerning the unfoldings relative to the above notions. The bifurcation set $B_{F}$ of $F$ is the set

$$
B_{F}=\left\{w \in \mathbb{R}^{r} \mid \frac{\partial F}{\partial w}=\frac{\partial^{2} F}{\partial w^{2}}=0 \text { at }(x, w)\right\} .
$$

The discriminant set of $F$ is the set $D_{F}=\left\{w \in \mathbb{R}^{r} \mid \frac{\partial F}{\partial w}=0\right.$ at $\left.(x, w)\right\}$. Then we have the following well-known result [4].

Theorem 5. Let $F:\left(I \times \mathbb{R}^{r},\left(x_{0}, w_{0}\right)\right) \rightarrow \mathbb{R}$ be an r-parameter unfolding of $f(x)$ which has $A_{k}-$ singularity at $x_{0}$.

(1) Suppose that $F$ is a (p)-versal unfolding.

(a) If $k=2$, then $B_{F}$ is locally diffeomorphic to $\{0\} \times \mathbb{R}^{r-1}$.

(b) If $k=3$, then $B_{F}$ is locally diffeomorphic to $C \times \mathbb{R}^{r-2}$.

(c) If $k=4$, then $B_{F}$ is locally diffeomorphic to $S W \times \mathbb{R}^{r-3}$.

(2) Suppose that $F$ is a versal unfolding.

(a) If $k=1$, then $D_{F}$ is locally diffeomorphic to $\{0\} \times \mathbb{R}^{r-1}$.

(b) If $k=2$, then $D_{F}$ is locally diffeomorphic to $C \times \mathbb{R}^{r-2}$.

(c) If $k=3$, then $D_{F}$ is locally diffeomorphic to $S W \times \mathbb{R}^{r-3}$.

Here, $C=\left\{\left(x_{1}, x_{2}\right): x_{1}^{2}=x_{2}^{3}\right\}$ is ordinary cusp and

$$
S W=\left\{\left(x_{1}, x_{2}, x_{3}\right): x_{1}=3 u^{4}+u^{2} v, x_{2}=4 u^{3}+2 u v, x_{3}=v\right\}
$$

is the swallowtail as indicated in Fig 1.

For the proof Theorem 1, we have the following key propositions. 
Proposition 6. Let $F_{h}: I \times S_{p G}^{2} \rightarrow \mathbb{R}$ be the pseudo-Galilean height function on a unit speed curve $\gamma(x)$. If $f_{h v_{0}}$ has $A_{k}$-singularity $(k=2,3)$ at $x_{0}$, then $F_{h}$ is a $(p)$-versal unfolding of $f_{h v_{0}}$.

Proof. We denote by $\gamma(x)=(x, y(x), z(x))$ and $v=\left(1, v_{2}, v_{3}\right)$. By definition, we have

$$
\begin{aligned}
F_{h}(x, \mathbf{v}) & =|t(x) n(x) \mathbf{v}| \\
& =\frac{1}{\kappa(x)}\left[y^{\prime \prime}(x) v_{3}-z^{\prime \prime}(x) v_{2}+y^{\prime}(x) z^{\prime \prime}(x)-y^{\prime \prime}(x) z^{\prime}(x)\right] .
\end{aligned}
$$

Let $J^{k-1}\left(\frac{\partial F_{h}}{\partial v_{i}}\left(x, v_{0}\right)\right)\left(x_{0}\right)$ be the $(k-1)$-jet of $\frac{\partial F_{h}}{\partial v_{i}}$ at $x_{0}(i=2,3)$; then we have

$$
J^{3}\left(\frac{\partial F_{h}}{\partial v_{i}}\left(x, v_{0}\right)\right)\left(x_{0}\right)=(-1)^{i+1}\left(b_{i}^{\prime}\left(x_{0}\right) x+\frac{1}{2} b_{i}^{\prime \prime}\left(x_{0}\right) x^{2}+\frac{1}{6} b_{i}^{\prime \prime \prime}\left(x_{0}\right) x^{3}\right),
$$

$i=2,3$. Here, $b(x)=\left(0, b_{2}, b_{3}\right)=\frac{1}{\kappa(x)}\left(0, z^{\prime \prime}(x), y^{\prime \prime}(x)\right)$ by the equation (5).

We distinguish two cases.

Case (1): $f_{h v_{0}}$ has the $A_{2}-$ singularity at $x_{0}$. We can define $1 \times 2$ matrix $A$ as follows:

$$
A=\left[\left(-\frac{z^{\prime \prime}\left(x_{0}\right)}{\kappa\left(x_{0}\right)}\right)^{\prime}\left(\frac{y^{\prime \prime}\left(x_{0}\right)}{\kappa\left(x_{0}\right)}\right)^{\prime}\right] .
$$

We also have $b^{\prime}\left(x_{0}\right)=\tau\left(x_{0}\right) n\left(x_{0}\right) \neq 0$ by the equation (6). Therefore we have $\operatorname{Rank} A=1$.

Case (2): $f_{h v_{0}}$ has the $A_{3}$-singularity at $x_{0}$. we define $2 \times 2$-matrix $B$ as follows:

$$
B=\left[\begin{array}{ll}
\left(-\frac{z^{\prime \prime}\left(x_{0}\right)}{\kappa\left(x_{0}\right)}\right)^{\prime} & \left(\frac{y^{\prime \prime}\left(x_{0}\right)}{\kappa\left(x_{0}\right)}\right)^{\prime} \\
\left(-\frac{z^{\prime \prime}\left(x_{0}\right)}{\kappa\left(x_{0}\right)}\right)^{\prime \prime} & \left(\frac{y^{\prime \prime}\left(x_{0}\right)}{\kappa\left(x_{0}\right)}\right)^{\prime \prime}
\end{array}\right]
$$

$B$ has to be nonsingular ( $\operatorname{det} B \neq 0$ ). By the Frenet-Serret formulas (5), we have the following calculation:

$$
\operatorname{det} B=-\left|t\left(x_{0}\right) \quad b^{\prime}\left(x_{0}\right) \quad b^{\prime \prime}\left(x_{0}\right)\right| .
$$

If the necessary derivatives of the Frenet-Serret formulas (6) is written, then we have $\operatorname{det} B=-\tau^{3}\left(x_{0}\right)$. Since $\tau(x) \neq 0$, the rank of $B$ is 2 .

Define the function $\widetilde{F}_{h}: I \times S_{p G}^{2} \times \mathbb{R} \rightarrow \mathbb{R}$ by $\widetilde{F}_{h}(x, \mathbf{v}, w)=F(x, \mathbf{v})-w$ and $f_{h \mathbf{v}, w}(x)=\widetilde{F}_{h}(x, \mathbf{v}, w)$. 
Proposition 7. If $f_{h \mathbf{v}_{0}, w_{0}}$ has $A_{k}$-singularity $(k=1,2,3)$ at $x_{0}$, then $F_{h}$ is a versal unfolding of $f_{h \mathbf{v}_{0}, w_{0}}$.

Proof. Using the same notations of Proposition 6, we have $\widetilde{F}_{h}\left(x, \mathbf{v}, v_{1}\right)=\frac{1}{\kappa(x)}\left[y^{\prime \prime}(x) v_{3}-z(x) v_{2}+y^{\prime}(x) z^{\prime \prime}(x)-y^{\prime \prime}(x) z^{\prime}(x)\right]-v_{1}$.

Let $J^{k-1}\left(\frac{\partial \widetilde{F}_{h}}{\partial v_{i}}\left(x, \mathbf{v}_{0}\right)\right)\left(x_{0}\right)$ be the $(k-1)-$ jet of $\frac{\partial \widetilde{F}_{h}}{\partial v_{i}}$ at $x_{0}(i=1,2,3)$; then we have

$$
\begin{aligned}
& \frac{\partial \widetilde{F}_{h}}{\partial v_{1}}\left(x_{0}, v_{0}\right)+J^{2}\left(\frac{\partial \widetilde{F}_{h}}{\partial v_{1}}\left(x, v_{0}\right)\right)\left(x_{0}\right)=-1 \\
& \frac{\partial \widetilde{F}_{h}}{\partial v_{i}}\left(x_{0}, v_{0}\right)+J^{2}\left(\frac{\partial \widetilde{F}_{h}}{\partial v_{i}}\left(x, v_{0}\right)\right)\left(x_{0}\right) \\
& =(-1)^{i+1}\left(b_{i}\left(x_{0}\right)+b_{i}^{\prime}\left(x_{0}\right) x+b_{i}^{\prime \prime}\left(x_{0}\right) \frac{x^{2}}{2}\right), i=2,3 .
\end{aligned}
$$

Now, we will distinguish three cases.

Case (1): $f_{h \mathbf{v}_{0}, w_{0}}$ has the $A_{1}-$ singularity at $x_{0}$. We can define $1 \times 2$ matrix $C$ as follows:

$$
C=\left[\begin{array}{lll}
-1 & \left(-\frac{z^{\prime \prime}\left(x_{0}\right)}{\kappa\left(x_{0}\right)}\right) & \left(\frac{y^{\prime \prime}\left(x_{0}\right)}{\kappa\left(x_{0}\right)}\right)
\end{array}\right] .
$$

The rank of $C$ is clearly 1 .

Case (2): $f_{h \mathbf{v}_{0}, w_{0}}$ has the $A_{2}$-singularity at $x_{0}$. we require the $2 \times 3$ matrix

$$
D=\left[\begin{array}{ccc}
-1 & \left(-\frac{z^{\prime \prime}\left(x_{0}\right)}{\kappa\left(x_{0}\right)}\right) & \left(\frac{y^{\prime \prime}\left(x_{0}\right)}{\kappa\left(x_{0}\right)}\right) \\
0 & \left(-\frac{z^{\prime \prime}\left(x_{0}\right)}{\kappa\left(x_{0}\right)}\right)^{\prime} & \left(\frac{y^{\prime \prime}\left(x_{0}\right)}{\kappa\left(x_{0}\right)}\right)^{\prime}
\end{array}\right]
$$

to have the maxsimal rank. By Case 1 in Proposition 6, the second line of $D$ does not vanish. Thus the rank of $D$ is 2 .

Case (3) : $f_{h v_{0}, w_{0}}$ has the $A_{3}-$ singularity at $x_{0}$. We can define the $3 \times 3$ matrix:

$$
E=\left[\begin{array}{ccc}
-1 & \left(-\frac{z^{\prime \prime}\left(x_{0}\right)}{\kappa\left(x_{0}\right)}\right) & \left(\frac{y^{\prime \prime}\left(x_{0}\right)}{\kappa\left(x_{0}\right)}\right) \\
0 & \left(-\frac{z^{\prime \prime}\left(x_{0}\right)}{\kappa\left(x_{0}\right)}\right)^{\prime} & \left(\frac{y^{\prime \prime}\left(x_{0}\right)}{\kappa\left(x_{0}\right)}\right)^{\prime} \\
0 & \left(-\frac{z^{\prime \prime}\left(x_{0}\right)}{\kappa\left(x_{0}\right)}\right)^{\prime \prime} & \left(\frac{y^{\prime \prime}\left(x_{0}\right)}{\kappa\left(x_{0}\right)}\right)^{\prime \prime}
\end{array}\right]
$$


to be non singular. By Case 2 in Proposition 6 , determinant of $E$ does not vanish. It means that the rank of $E$ is 3 .

For the pseudo-Galilean distance function $F_{d}$, we have the following proposition.

Proposition 8. Let $F_{d}: I \times G_{3}^{1} \rightarrow \mathbb{R}$ be the pseudo-Galilean distance function on a unit speed curve $\gamma(x)$. If $f_{d u_{0}}$ has $A_{k}-$ singularity $(k=2,3,4)$ at $x_{0}$, then $F_{d}$ is a $(p)$-versal unfolding of $f_{d u_{0}}$.

Proof. Denote $\gamma(x)=(x, y(x), z(x))$ and $\mathbf{u}=\left(u_{1}, u_{2}, u_{3}\right)$. Then by definition, we have

$$
\begin{aligned}
& F_{d}(x, \mathbf{u})=|t(x) n(x) \gamma(x)-\mathbf{u}| \\
& =\left(x-u_{1}\right)\left(y^{\prime}(x) \frac{z^{\prime \prime}(x)}{\kappa(x)}-z^{\prime}(x) \frac{y^{\prime \prime}(x)}{\kappa(x)}\right) \\
& -\left(y(x)-u_{2}\right) \frac{z^{\prime \prime}(x)}{\kappa(x)}+\left(z(x)-u_{3}\right) \frac{y^{\prime \prime}(x)}{\kappa(x)} \text {. }
\end{aligned}
$$

Let $J^{k-1}\left(\frac{\partial F_{d}}{\partial u_{i}}\left(x, u_{0}\right)\right)\left(x_{0}\right)$ be the $(k-1)$-jet of $\frac{\partial F_{d}}{\partial u_{i}}$ at $x_{0}$. If we denote that $p(x)=-y^{\prime}(x) \frac{z^{\prime \prime}(x)}{\kappa(x)}+z^{\prime}(x) \frac{y^{\prime \prime}(x)}{\kappa(x)}$. Then we have

$$
\begin{aligned}
& J^{3}\left(\frac{\partial F_{d}}{\partial u_{1}}\left(x, u_{0}\right)\right)\left(x_{0}\right)=p^{\prime}\left(x_{0}\right) x+\frac{1}{2} p^{\prime \prime}\left(x_{0}\right) x^{2}+\frac{1}{6} p^{\prime \prime \prime}\left(x_{0}\right) x^{3} \\
& J^{3}\left(\frac{\partial F_{d}}{\partial u_{i}}\left(x, u_{0}\right)\right)\left(x_{0}\right)=(-1)^{i}\left(b_{i}^{\prime}\left(x_{0}\right) x+\frac{1}{2} b_{i}^{\prime \prime}\left(x_{0}\right) x^{2}+\frac{1}{6} b_{i}^{\prime \prime \prime}\left(x_{0}\right) x^{3}\right), i=2,3 .
\end{aligned}
$$

Here, $b(x)=\left(0, b_{2}, b_{3}\right)=\frac{1}{\kappa(x)}\left(0, z^{\prime \prime}(x), y^{\prime \prime}(x)\right)$ by the equation $(5)$.

We distinguish three cases.

Case (1): $f_{d u_{0}}$ has the $A_{2}$-singularity at $x_{0}$. We can define a $1 \times 3$ matrix $G$ as follows:

$$
\begin{aligned}
G= & {\left[\begin{array}{lll}
p^{\prime}\left(x_{0}\right) & b_{2}^{\prime}\left(x_{0}\right) & -b_{3}^{\prime}\left(x_{0}\right)
\end{array}\right] } \\
& {\left[\left(y^{\prime}\left(x_{0}\right) \frac{z^{\prime \prime}\left(x_{0}\right)}{\kappa\left(x_{0}\right)}-z^{\prime}\left(x_{0}\right) \frac{y^{\prime \prime}\left(x_{0}\right)}{\kappa\left(x_{0}\right)}\right)^{\prime}\left(\frac{z^{\prime \prime}\left(x_{0}\right)}{\kappa\left(x_{0}\right)}\right)^{\prime}\left(-\frac{y^{\prime \prime}\left(x_{0}\right)}{\kappa\left(x_{0}\right)}\right)^{\prime}\right] . }
\end{aligned}
$$

We have $b^{\prime}\left(x_{0}\right)=\tau\left(x_{0}\right) n\left(x_{0}\right) \neq 0$ by the equation (6). This means that $\operatorname{Rank} G=1$. 
Case (2): $f_{d u_{0}}$ has the $A_{3}$-singularity at $x_{0}$. We can define a $2 \times 3$ matrix $H$ as follows:

$$
H=\left[\begin{array}{lll}
p^{\prime}\left(x_{0}\right) & b_{2}^{\prime}\left(x_{0}\right) & -b_{3}^{\prime}\left(x_{0}\right) \\
p^{\prime \prime}\left(x_{0}\right) & b_{2}^{\prime \prime}\left(x_{0}\right) & -b_{3}^{\prime \prime}\left(x_{0}\right)
\end{array}\right]
$$

In Case 1 in Proposition 6, we get that the rank of $H$ is 2.

Case (3): $f_{d u_{0}}$ has the $A_{4}$-singularity at $x_{0}$. We can define a $3 \times 3$ matrix:

$$
K=\left[\begin{array}{ccc}
p^{\prime}\left(x_{0}\right) & b_{2}^{\prime}\left(x_{0}\right) & -b_{3}^{\prime}\left(x_{0}\right) \\
p^{\prime \prime}\left(x_{0}\right) & b_{2}^{\prime \prime}\left(x_{0}\right) & -b_{3}^{\prime \prime}\left(x_{0}\right) \\
p^{\prime \prime \prime}\left(x_{0}\right) & b_{2}^{\prime \prime \prime}\left(x_{0}\right) & -b_{3}^{\prime \prime \prime}\left(x_{0}\right)
\end{array}\right]
$$

to be nonsingular. It is enough to show that $|K| \neq 0$. To this end, one can use direct calculations. Instead of this, we will choose a simpler way. Let $\mathbf{e}=(1,0,0)$. Then we have $p(x)=\mid t(x)$ e $n(x) \mid$ and

$$
\begin{aligned}
p^{\prime}(x)= & \tau(x) \mid t(x) \text { e } b(x) \mid \\
p^{\prime \prime}(x)= & \tau^{\prime}(x) \mid t(x) \text { e } b(x)\left|+\tau^{2}(x)\right| t(x) \text { e } n(x) \mid-\kappa(x) \tau(x) \\
p^{\prime \prime \prime}(x)= & \left(\tau^{\prime \prime}(x)+\tau^{3}(x)\right) \mid t(x) \text { e } b(x)\left|+3 \tau(x) \tau^{\prime}(x)\right| t(x) \text { e } n(x) \mid \\
& -\kappa^{\prime}(x) \tau(x)-2 \kappa(x) \tau^{\prime}(x)
\end{aligned}
$$

which are calculated according to the first column of the matrix $K$. Therefore we have

$$
\begin{aligned}
|K|= & p^{\prime}\left(x_{0}\right)\left|\mathbf{e} n^{\prime \prime}\left(x_{0}\right) n^{\prime \prime \prime}\left(x_{0}\right)\right|-p^{\prime \prime}\left(x_{0}\right)\left|\mathbf{e} n^{\prime}\left(x_{0}\right) n^{\prime \prime \prime}\left(x_{0}\right)\right| \\
& +p^{\prime \prime \prime}\left(x_{0}\right)\left|\mathbf{e} n^{\prime}\left(x_{0}\right) n^{\prime \prime}\left(x_{0}\right)\right|
\end{aligned}
$$

Here, if we write necessary derivatives of the Frenet-Serret formulas in (7), then we have $|K|=-\kappa^{2}\left(x_{0}\right) \tau^{3}\left(x_{0}\right)\left(\frac{\tau}{\kappa}\right)^{\prime}\left(x_{0}\right)$. By the assumptions $\kappa \neq 0, \tau \neq 0$ and $f_{d u_{0}}$ has $A_{4}$-singularity at $x_{0}$, we have $\left(\frac{\tau}{\kappa}\right)^{\prime}\left(x_{0}\right) \neq 0$, and so $|K| \neq 0$.

Corollary 9. Proof of Theorem 1 follows from, Propositions 2, 3, 6, 7, 8 and Theorem 5. By Proposition 2, we know that the bifurcations set of $F_{h}(x, \mathbf{v})$ is pseudo-Galilean spherical Darboux image, by Theorem 5 and Proposition 6, Theorem 1(1) holds. By Proposition 2, we know that the discriminant set of $\widetilde{F}_{h}(x, \mathbf{v}, w)$ is diffeomorphic to the rectifying Gaussian surface, by Theorem 5 and Proposition 7, Theorem 1(2) holds. By Proposition 3 , we know that the bifurcation set of $F_{d}(x, \mathbf{u})$ is the rectifying developable surface, Theorem 5 and Proposition 8, Theorem 1(3) holds. 
Example 10. Consider the curve $\gamma: I \subset \mathbb{R} \rightarrow E^{3}, \gamma(x)=\left(x, \frac{x^{2}}{\sqrt{2}}, \frac{x^{3}}{3}\right)$.

For a arbitrary speed curve $\gamma: I \rightarrow E^{3}, I \subset \mathbb{R}$ the associated moving trihedron is given by

$$
T(x)=\frac{\gamma^{\prime}(x)}{\left\|\gamma^{\prime}(x)\right\|}, \quad B(x)=\frac{\gamma^{\prime}(x) \times \gamma^{\prime \prime}(x)}{\left\|\gamma^{\prime}(x) \times \gamma^{\prime \prime}(x)\right\|}, N(x)=B(x) \times T(x)
$$

and the curvature $\kappa(x)$ and the torsion $\tau(x)$ are defined by

$$
\kappa(x)=\frac{\left\|\gamma^{\prime}(x) \times \gamma^{\prime \prime}(x)\right\|}{\left\|\gamma^{\prime}(x)\right\|^{3}}, \tau(x)=\frac{\operatorname{det}\left(\gamma^{\prime}(x), \gamma^{\prime \prime}(x), \gamma^{\prime \prime \prime}(x)\right)}{\kappa^{2}(x)} .
$$

The vectors $T(x), N(x)$ and $B(x)$ are called the vectors of the tangent, principal normal and the binormal line, respectively [14].

We compute the Frenet apparatus of the curve $\gamma(x)=\left(x, \frac{x^{2}}{\sqrt{2}}, \frac{x^{3}}{3}\right)$. If the necessary derivatives of the Frenet-Serret formulas is written, then we have $T(x)=\frac{1}{1+x^{2}}\left(1, \sqrt{2} x, x^{2}\right), \quad B(x)=\frac{1}{1+x^{2}}\left(x^{2},-\sqrt{2} x, 1\right)$, $N(x)=\frac{1}{1+x^{2}}\left(-\sqrt{2} x, 1-x^{2}, \sqrt{2} x\right), \kappa(x)=\frac{1}{2 \cosh ^{2} x}, \tau(x)=4 \cosh ^{4} x$.

Therefore we compute $R D(\gamma)=\{\gamma(x)+u \widetilde{D}(x) \mid u \in \mathbb{R}, x \in I\}$, here a curve by $\widetilde{D}(x)=\left(-\frac{\tau}{\kappa}\right)(x) T(x)+B(x)$. Hence, we have

$$
R D(\gamma)=\left(x+\frac{u\left(x^{2}-1\right)}{1+x^{2}}, \frac{x^{2}}{\sqrt{2}}-\frac{u(2 \sqrt{2} x)}{1+x^{2}}, \frac{x^{3}}{3}+\frac{u\left(1-x^{2}\right)}{1+x^{2}}\right) .
$$

We also consider the curve $\gamma: I \subset \mathbb{R} \rightarrow G_{1}^{3}, \gamma(x)=\left(x, \frac{x^{2}}{\sqrt{2}}, \frac{x^{3}}{3}\right)$. If the necessary derivatives of the Frenet-Serret formulas (5) is written, then we have

$$
\begin{aligned}
t(x) & =\left(1, \sqrt{2} x, x^{2}\right), n(x)=\frac{1}{\sqrt{2+4 x^{2}}}(0, \sqrt{2}, 2 x), \\
b(x) & =\frac{1}{\sqrt{2+4 x^{2}}}(0,2 x, \sqrt{2}) \\
\kappa(x) & =\sqrt{2+4 x^{2}}, \tau(x)=\frac{\sqrt{2}}{1+2 x^{2}} .
\end{aligned}
$$

It follows that

$$
R D(\gamma)=\left(x-\frac{u}{\left(1+2 x^{2}\right)^{\frac{3}{2}}}, \frac{x^{2}}{\sqrt{2}}-\frac{u\left(2 \sqrt{2} x^{3}\right)}{\left(1+2 x^{2}\right)^{\frac{3}{2}}}, \frac{x^{3}}{3}+\frac{u\left(1+x^{2}\right)}{\left(1+2 x^{2}\right)^{\frac{3}{2}}}\right) \text {. }
$$



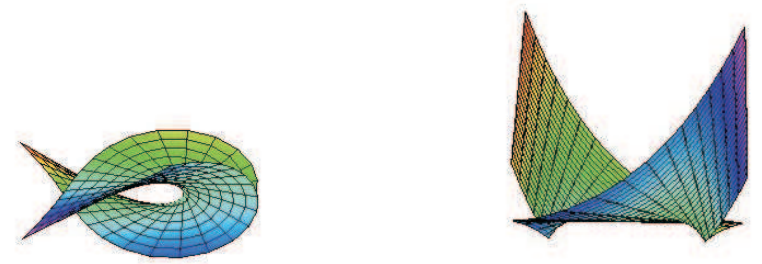

Figure 2: The Euclidean rectifying developable surface and the pseudoGalilean rectifying developable surface

Acknowledgement. The authors would like to thank the referee for the helpful suggestions.

\section{REFERENCES}

1. Arnol'D, V.I.; Gusĕñ-Zade, S.M.; Varchenko, A.N. - Singularities of Differentiable Maps, vol. I. The classification of critical points, caustics and wave fronts. Translated from the Russian by Ian Porteous and Mark Reynolds. Monographs in Mathematics, 82, Birkhäuser Boston, Inc., Boston, MA, 1985.

2. BRuce, J.W. - On singularities, envelopes and elementary differential geometry, Math. Proc. Cambridge Philos. Soc., 89 (1981), 43-48.

3. Bruce, J.W.; Giblin, P.J. - Generic geometry, Amer. Math. Monthly, 90 (1983), $529-545$.

4. Bruce, J.W.; Giblin, P.J. - Curves and Singularities, A geometrical introduction to singularity theory. Second edition, Cambridge University Press, Cambridge, 1992.

5. Che, M.G.; Jiang, Y.; Pei, Dong He - The hyperbolic Darboux image and rectifying Gaussian surface of nonlightlike curve in Minkowski 3-space, J. Math. Res. Exposition, 28 (2008), 651-658.

6. Divjak, B. - Geometrija psudogalilejevih prostora, Ph.D. Thesis, University of Zagreb, 1997.

7. Divjak, B.; Milin ŠıPuš, Ž. - Some special surfaces in the pseudo-Galilean space, Acta Math. Hungar., 118 (2008), 209-226.

8. Erjavec, Z.; Divjak, B. - The equiform differential geometry of curves in the pseudo-Galilean space, Math. Commun., 13 (2008), 321-332. 
9. Izumiya, S.; Katsumi, H.; Yamasaki, T. - The rectifying developable and the spherical Darboux image of a space curve, Geometry and topology of caustics-CAUSTICS '98 (Warsaw), 137-149, Banach Center Publ., 50, Polish Acad. Sci. Inst. Math., Warsaw, 1999.

10. Izumiya, S.; Sano, T. - Generic affine differential geometry of space curves, Proc. Roy. Soc. Edinburgh Sect. A, 128 (1998), 301-314.

11. MolnáR, E. - The projective interpretation of the eight 3-dimensional homogeneous geometries, Beiträge Algebra Geom., 38 (1997), 261-288.

12. Mond, D. - Singularities of the tangent developable surface of a space curve, Quart. J. Math. Oxford Ser., 40 (1989), 79-91.

13. Montaldi, J.A. - On contact between submanifolds, Michigan Math. J., 33 (1986), 195-199.

14. O’Neill, B. - Elementary Differential Geometry, Academic Press, New YorkLondon, 1966.

15. PEI, D.; SANO, T. - The focal developable and the binormal indicatrix of a nonlightlike curve in Minkowski 3-space, Tokyo J. Math., 23 (2000), 211-225.

16. Röschel, O. - Die Geometrie des Galileischen Raumes, [The geometry of Galilei space] Berichte [Reports], 256, Forschungszentrum Graz, Mathematisch-Statistische Sektion, Graz, 1985.

17. Yaglom, I.M. - A Simple Non-Euclidean Geometry and its Physical Basis, SpringerVerlag, New York-Heidelberg, 1979.

Received: 27.I.2012

Revised: 30.IV.2012

Accepted: 16.V.2012
Amasya University, Faculty of Sciences and Arts, Department of Mathematics,

Amasya, TURKEY

tevfiksah@gmail.com

Ondokuz Mayis University, Faculty of Sciences and Arts, Department of Mathematics, Samsun, TURKEY murtezay@omu.edu.tr 